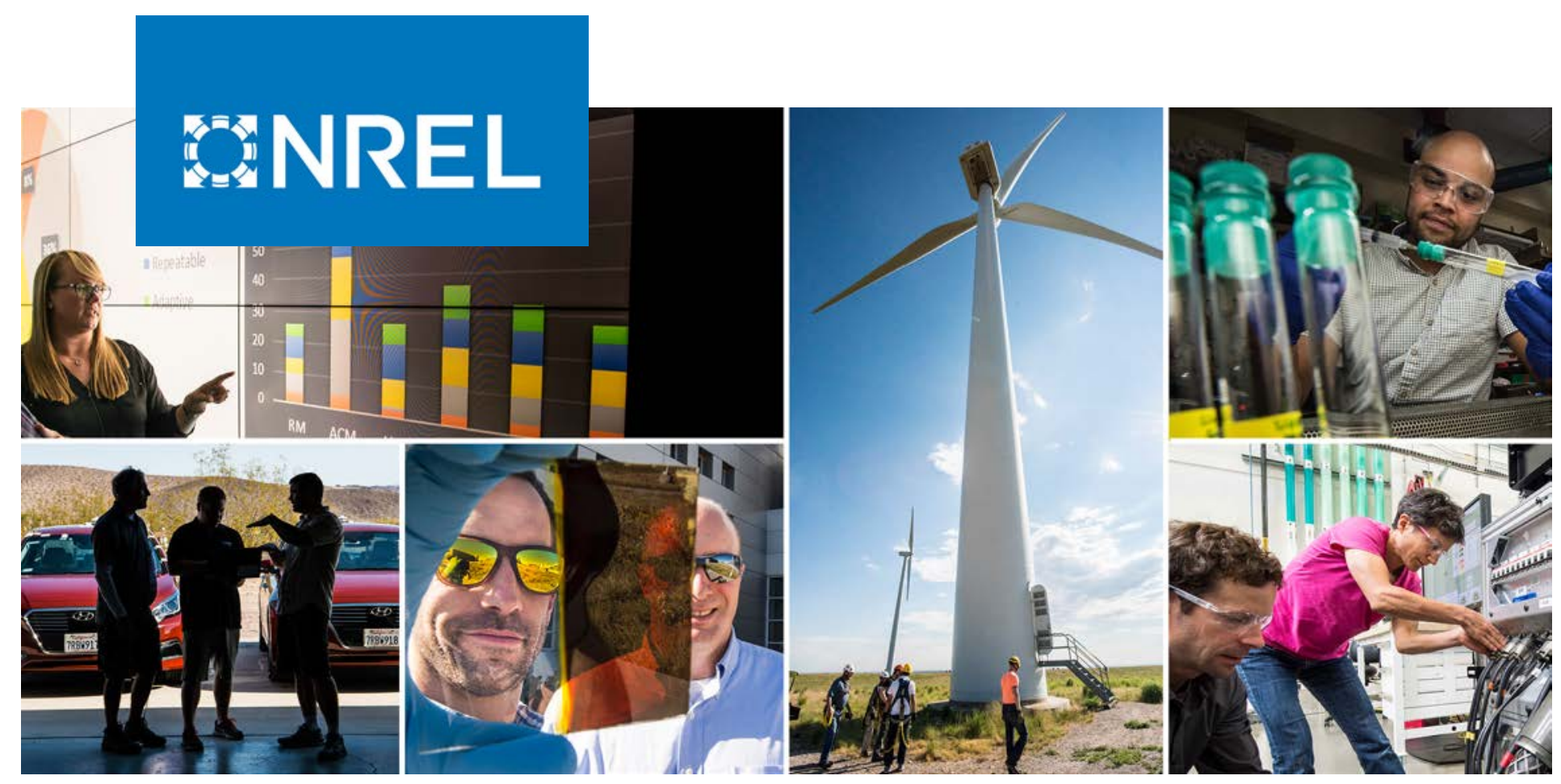

\title{
Impact of Inverter-Interfaced Renewable Generation on Transient Stability at Varying Levels of Penetration
}

\section{Preprint}

Zhao Liu, ${ }^{1}$ Ziang Zhang, ${ }^{1}$ and Yashen Lin ${ }^{2}$

${ }^{1}$ Binghamton University

${ }^{2}$ National Renewable Energy Laboratory

Presented at the IEEE Annual Conference of the IEEE Industrial Electronics Society (IECON)

Washington, D.C.

October 21-23, 2018

NREL is a national laboratory of the U.S. Department of Energy

Office of Energy Efficiency \& Renewable Energy

Operated by the Alliance for Sustainable Energy, LLC

This report is available at no cost from the National Renewable Energy Laboratory (NREL) at www.nrel.gov/publications.

\section{Conference Paper}

NREL/CP-5D00-70489

March 2019 


\title{
GHREL
}

Impact of Inverter-Interfaced

Renewable Generation on Transient

Stability at Varying Levels of

Penetration

\section{Preprint}

\author{
Zhao Liu, ${ }^{1}$ Ziang Zhang, ${ }^{1}$ and Yashen Lin ${ }^{2}$ \\ 1 Binghamton University \\ ${ }^{2}$ National Renewable Energy Laboratory
}

\section{Suggested Citation}

Liu, Zhao, Ziang Zhang, and Yashen Lin. 2019. Impact of Inverter-Interfaced Renewable Generation on Transient Stability at Varying Levels of Penetration: Preprint. Golden, CO: National Renewable Energy Laboratory. NREL/CP-5D00-70489.

https://www.nrel.gov/docs/fy19osti/70489.pdf.

\begin{abstract}
(c) 2019 IEEE. Personal use of this material is permitted. Permission from IEEE must be obtained for all other uses, in any current or future media, including reprinting/republishing this material for advertising or promotional purposes, creating new collective works, for resale or redistribution to servers or lists, or reuse of any copyrighted component of this work in other works.
\end{abstract}

NREL is a national laboratory of the U.S. Department of Energy Office of Energy Efficiency \& Renewable Energy Operated by the Alliance for Sustainable Energy, LLC

This report is available at no cost from the National Renewable Energy Laboratory (NREL) at www.nrel.gov/publications.

Contract No. DE-AC36-08GO28308
Conference Paper

NREL/CP-5D00-70489

March 2019

National Renewable Energy Laboratory 15013 Denver West Parkway Golden, CO 80401

303-275-3000 • www.nrel.gov 


\section{NOTICE}

This work was authored in part by the National Renewable Energy Laboratory, operated by Alliance for Sustainable Energy, LLC, for the U.S. Department of Energy (DOE) under Contract No. DE-AC36-08GO28308. Funding provided by U.S. Department of Energy Office of Energy Efficiency and Renewable Energy Solar Energy Technologies Office. The views expressed herein do not necessarily represent the views of the DOE or the U.S. Government. The U.S. Government retains and the publisher, by accepting the article for publication, acknowledges that the U.S. Government retains a nonexclusive, paid-up, irrevocable, worldwide license to publish or reproduce the published form of this work, or allow others to do so, for U.S. Government purposes.

This report is available at no cost from the National Renewable Energy Laboratory (NREL) at www.nrel.gov/publications.

U.S. Department of Energy (DOE) reports produced after 1991 and a growing number of pre-1991 documents are available free via www.OSTI.gov.

Cover Photos by Dennis Schroeder: (clockwise, left to right) NREL 51934, NREL 45897, NREL 42160, NREL 45891, NREL 48097, NREL 46526.

NREL prints on paper that contains recycled content. 


\section{Impact of Inverter-Interfaced Renewable Generation on Transient Stability at Varying Levels of Penetration}

\author{
Zhao Liu, Ziang Zhang \\ Department of Electrical and Computer Engineering \\ The State University of New York at Binghamton \\ Binghamton, NY, USA \\ \{zliu86, ziang.zhang\}@binghamton.edu
}

\author{
Yashen Lin \\ Power System Engineering Center \\ National Renewable Energy Laboratory \\ Golden, CO, USA \\ Yashen.Lin@nrel.gov
}

\begin{abstract}
The transient dynamics of conventional power systems are dominated by synchronous generators. Because inverters serve as the primary interface between the power systems and low-inertia renewable energy resources, increasing penetration of such inverter-interfaced generation has a growing impact on the transient dynamics of power systems. In this paper, we attempt to quantify such an impact at different penetration levels. An elementary system, which includes one scalable inverter, one synchronous generator and one load, is studied under different parameter settings to assess the transient dynamics of the system. To quantify the transient stability, the post-fault Region of Attraction (ROA) of the system is estimated by the sample-based basin stability approach with parallel computing technology. Simulation results suggest that different inverter penetration levels, inertias of the generator, inverter-controller gains and phase-locked loop controller-gains have a clear impact of the ROA as well as the transient dynamics of the elementary systems. Increasing the penetration level of inverter-interfaced generations will decrease the volume of ROA of generators, which is also verified with a modified IEEE 9-bus system model.
\end{abstract}

Index Terms--Transient stability, inverter-interfaced generation, renewable energy integration, basin stability, parallel computing.

\section{INTRODUCTION}

Power generation is evolving from conventional centralized fossil fuel generation to distributed renewable resources. The inertia of synchronous generators can absorb some energy caused by power system faults or other disturbances, which is the key to the stability of current power systems. As the ratio of renewable energy resource increases, the dynamic behavior of the power system has been gradually changing. Studies have raised concerns that the power system is losing resilience as the system loses inertia [1]. In this paper, we attempt to quantify the system transient stability as we transition from conventional generator-dominated systems to systems with significant inverter-interfaced renewable energy resources.

We investigate an elementary system in this work in Fig. 1, which consists of one 555MW conventional generator, one inverter-interfaced generator, and one load. Although it is

This work was authored in part by Alliance for Sustainable Energy, LLC, the Manager and Operator of the National Renewable Energy Laboratory for the U.S. Department of Energy (DOE) under Contract No. DE-AC3608GO28308. tempting to study a more practical system with large collections of interconnected synchronous generators and inverters, understanding the dynamics of a single pair of generators and inverters in an elementary system is an inevitable first step for future studies of complex systems. The impact of the inverter penetration is also verified with a modified IEEE 9-bus system model. A scalable-lumped inverter model is used to represent a large number of parallel inverters with heterogeneous power output ratings [2]. The parameter scaling framework allows us to systematically scale all the model parameters, filter elements and controller gains, and to investigate different inverter penetration levels [2], [3]. This work is focused on assessing the impacts and concerns under the business-as-usual approaches, which means the current grid-following control strategy is assigned to the inverter. Other advanced inverter control strategies proposed in the literature are beyond the scope of this paper.

Many previous works on this topic focused on the impact of inverter-interfaced generation on distribution feeders or microgrid where the dynamics of conventional generators are ignored, and the studied systems are connected to an infinite bus at the point of common coupling. The voltage stability issue has been studied in [4], [5]. Authors in [5] also compared impacts of a distributed inverter-interfaced PV system versus a centralized inverter-interfaced PV system on the bulk power system. The small-signal stability has been studied at varying levels of penetrations in [3], with different inverter and generator placement in [6], and with AC/DC hybrid microgrid in [7]. However, the transient stability with varying levels of penetrations on a similar system has not been explicitly addressed. One of the main barriers to understanding the transient stability of such systems is the complex nonlinear behavior of synchronous generators, inverters, and the interaction among these devices. Thus, a transient stability

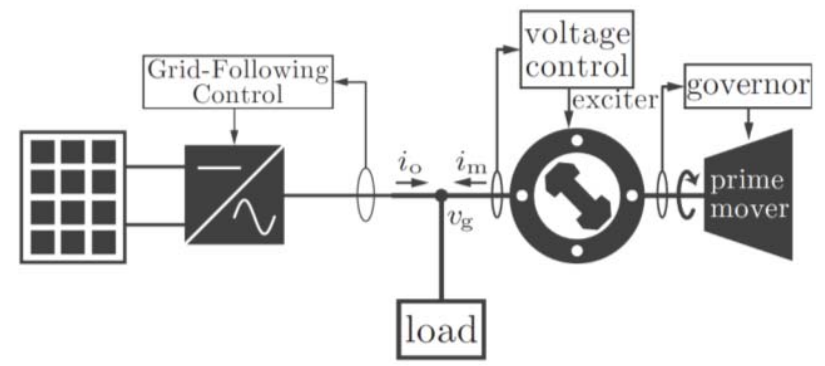

Fig. 1. An elementary system model. 


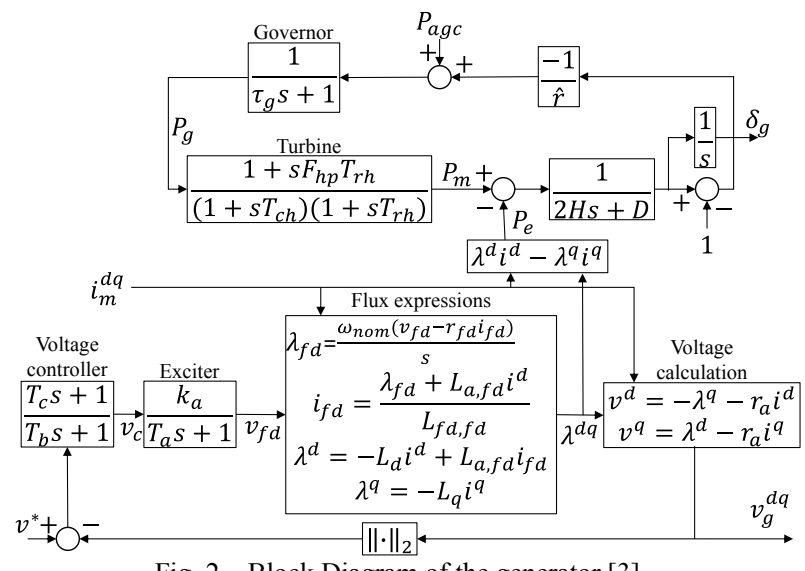

Fig. 2. Block Diagram of the generator [3].

analysis approach that can work with high fidelity models is desired.

To quantify the transient stability in general, many studies have been done under power system probabilistic frameworks. In these studies [8]-[12], the Monte Carlo simulation method was commonly used to sample the binary states of devices (Normal or Outage), the probability distributions of fault types, locations, durations, and the probability distributions of uncertainties of renewable generations to generate corresponding metrics. The results of these metrics rely heavily on the accurate assumptions of the above probabilities for which it is usually difficult to find enough historical data and formulate [11], and are limited to the fault types and uncertainty scenarios considered in the assumptions.

In this work, a basin stability method is used to quantify the transient stability of the test system. The basin stability concept was originally introduced by researchers in the physics community to quantify the stability of coupled oscillators [13] and nonlinear systems [14]-[17], which was done by measuring the probability that these systems remain stable after disturbances. Different from other probability frameworks, the basin stability method focuses on directly sampling the postfault state space of the dynamic states of the system according to a predesigned range and distribution, and justifies the stability of each sample with time-domain simulations. The result depends more on the dynamic behaviors of the system itself at post-fault, which is related to the volume of the ROA (Region of Attraction). In addition, because the basin stability is a simulation-based method, it is easily applicable to nonlinear, high-order power system models that include inverters and controllers. Long simulation time is one of the major disadvantages of such simulation-based approaches. In this work, the state-of-the-art parallel computing architecture is used to speed up the basin stability analysis. The basin stability method has been successfully applied to analyze the transient stabilities of several detailed power system models with synchronous generators [18], [19].

The contributions of this work are 1) analysis of the transient stability impact of adding inverter-interfaced generations to conventional systems with different penetration levels, 2) application of a basin stability method to quantify the transient stability of such systems with the help of parallel computing technology, 3) investigation of the impact of system

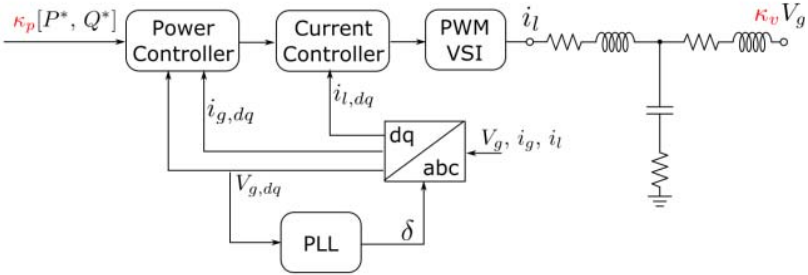

Fig. 3. Block diagram of the scalable inverter [3].

parameters on system stability, including inertias of generators, inverter's current controller gains and PLL (phase-locked loop) controller gains.

\section{THE POWER SYSTEM MODEL}

\section{A. Sychronous generator model}

We use an eighth-order steam-driven generator model in this work in Fig. 2. It includes a synchronous machine, a steam turbine, a governor, a voltage controller and an exciter. Considering the synchronous generator model is already well established, only a brief description of the model is given in this section. Detailed equations and parameters of this system can be found in [20]. This system can be summarized with the following state-space representation:

$$
\dot{x}_{m}=f_{m}\left(x_{m}, u_{m}\right) \text {, }
$$

where the system states $x_{m}$ and inputs $u_{m}$ are defined as

$$
\begin{gathered}
x_{m}=\left[\delta_{m}, \omega_{m}, P_{g}, P_{t}, P_{m}, v_{c}, v_{f d}, \lambda_{f d}\right]^{T}, \\
u_{m}=\left[P_{a g c}, v^{*}, i_{m}^{d q}\right]^{T},
\end{gathered}
$$

The swing equation, steam turbine and governor form the frequency dynamic loop of the generator. $P_{a g c}$ is the input reference of power generation from the automatic generation control. $P_{g}, P_{t}$, and $P_{m}$ are governor output, internal state of the turbine and mechanical power input of the swing equation, respectively. The generator terminal voltage is controlled by a type AC4A [20] exciter with a lead-lag compensator. $v_{c}, v_{f d}$ and $\lambda_{f d}$ are voltage controller output, field voltage and field flux linkage of the generator, respectively. The terminal voltage magnitude reference is denoted as $v^{*}$. The references of terminal currents in d-/q-axis are $i_{m}^{d}, i_{m}^{q}$. Higher-order dynamics of the generator are neglected in the current stage of analysis.

\section{B. Scalable inverter model}

To appropriately model the inverters at different penetration levels in the system, a scalable-lumped inverter model is used in this study. Fig. 3 shows the structure of this model, which includes a LCL filter, a PLL, a power controller and a current controller. Due to the page limits of this paper, detailed equations of the controllers can be found in [3]. The PWM controller and three-leg voltage source inverter are represented with an averaged model. Similar to the synchronous machine model, the inverter model before the scaling can be written as

$$
\dot{x}_{i}=f_{i}\left(x_{i}, u_{i}\right) \text {, }
$$

where the system states $x_{i}$ and inputs $u_{i}$ are defined as 


$$
\begin{gathered}
x_{i}=\left[i_{l}^{d q}, i_{o}^{d q}, v_{o}^{d q}, \gamma^{d q}, p_{a v g}, q_{a v g}, \emptyset_{p q}, v_{P L L}, \emptyset_{P L L}, \delta_{i}\right]^{T}, \\
u_{i}=\left[p^{*}, q^{*}, v_{g}^{d q}\right]^{T} .
\end{gathered}
$$

The $i_{l}^{d q}$ and $i_{o}^{d q}$ are the inverter side and grid side current of the LCL filter in d-/q-axis. The output voltage of the LCL filter is donated as $v_{o}^{d q}$. $\gamma^{d q}$ and $\emptyset_{p q}$ are the internal states of the current and power PI controller. $p_{\text {avg }}$ and $q_{\text {avg }}$ are measured real and reactive power through a low-pass filter in the power controller. $v_{P L L}$ is the measured d-axis voltage as input of the PLL and $\emptyset_{P L L}$ is the compensator state of the PLL. $\delta_{i}$ represents the operating angle of the inverter. The input signals $p^{*}$ and $q^{*}$ are the real and reactive power set points, and $v_{g}^{d q}$ is the grid voltage at the terminal point.

The original parameters of this inverter are measured from a custom-built hardware prototype at NREL whose power rating is $P_{i}=1 \mathrm{~kW}$ and line to neutral voltage rating is $v_{i}=$ $120 \mathrm{~V}$. For scaling the inverter, a scaling law is introduced with a power tuning coefficient $\kappa_{p}$ and voltage tuning coefficient $\kappa_{v}$ [2], [3]. Suppose the scaled inverter has a power rating $\bar{P}=$ $\kappa_{p} P_{i}$ and voltage rating $\bar{v}=\kappa_{v} v_{i}$. The inverter inputs are scaled accordingly as $u_{i}^{S}=\left[\kappa_{p} p^{*}, \kappa_{p} q^{*}, \kappa_{v} v_{g}^{d q}\right]^{T}$. Then the states of the scaled inverter $x_{i}^{S}(t)$ are a scaled version of the original inverter state $x_{i}(t)$ by a tuning vector $\underline{\mathrm{K}}$, i.e.,

$$
\begin{gathered}
x_{i}^{S}(t)=\operatorname{diag}(\underline{\mathrm{\kappa}}) x_{i}(t), \forall t \geq t_{0} \\
\underline{\kappa}=\left[\frac{\mathrm{\kappa}_{p}}{\mathrm{\kappa}_{v}}, \frac{\mathrm{\kappa}_{p}}{\mathrm{\kappa}_{v}}, \mathrm{\kappa}_{v}, \frac{\mathrm{\kappa}_{p}}{\mathrm{\kappa}_{v}}, \mathrm{\kappa}_{p}, \mathrm{\kappa}_{p}, \mathrm{\kappa}_{p}, \mathrm{\kappa}_{v}, \mathrm{\kappa}_{v}, 1\right]^{T} .
\end{gathered}
$$

Further, the PI controller gains $K_{P}$ and $K_{I}$ of power, current controller and PLL also need to be tuned as below in Table I. Other parameters of the inverter are scaled according to [3].

TABLE I. ORIGINAL VALUES AND SCALING LAWS OF CONTROLLERS GAINS

\begin{tabular}{|c|c|c|c|c|}
\hline Controller & \multicolumn{2}{|c|}{ Proportional gain $K_{\boldsymbol{P}}$} & \multicolumn{2}{|c|}{ Integral gain $\boldsymbol{K}_{\boldsymbol{I}}$} \\
\hline & $\begin{array}{c}\text { Original } \\
\text { Values }\end{array}$ & $\begin{array}{c}\text { Scaling } \\
\text { Laws }\end{array}$ & $\begin{array}{c}\text { Original } \\
\text { Values }\end{array}$ & $\begin{array}{c}\text { Scaling } \\
\text { Laws }\end{array}$ \\
\hline Current & $16.4 \mathrm{~V} / \mathrm{A}$ & $\left(\mathrm{\kappa}_{v}^{2} / \mathrm{\kappa}_{p}\right) K_{P}$ & $30.4 \mathrm{~V} /(\mathrm{A} \cdot \mathrm{s})$ & $\left(\mathrm{\kappa}_{v}^{2} / \mathrm{\kappa}_{p}\right) K_{I}$ \\
\hline Power & $0.01(\mathrm{~V})^{-1}$ & $K_{P} / \mathrm{\kappa}_{v}$ & $0.1(\mathrm{~V} \cdot \mathrm{s})^{-1}$ & $K_{I} / \mathrm{\kappa}_{v}$ \\
\hline PLL & $0.25 \mathrm{rad} / \mathrm{V}$ & $K_{P} / \mathrm{\kappa}_{v}$ & $2 \mathrm{rad} /(\mathrm{V} \cdot \mathrm{s})$ & $K_{I} / \mathrm{\kappa}_{v}$ \\
\hline
\end{tabular}

From the system point of view, increasing the tuning parameters $\kappa_{p}$ and $\kappa_{v}$ are equivalent to add more small parallel connected inverters into the system or replace the smaller inverters with larger inverters [2]. With the help of the above scaling process, the inverter can be systematically scaled to change the ratings and simulate different levels of penetrations of inverter-interface generations.

\section{Network model}

For this simple elementary system, the network model can be defined as

$$
v_{g}=z\left(i_{i}+i_{g}\right)
$$

where $v_{g}$ is the voltage of the grid, $z$ is the complex load impedance, $i_{i}$ and $i_{g}$ are the currents from inverter and generator, respectively. Notice that the currents from the inverter and the generator need to take a $d q$ reference frame transformation before substitution into equation (9) [3].

\section{THE BASIN STABILITY METHOD}

In power systems, when a fault or other types of disturbance happens, the generator's operating point will be pushed away from the stable equilibrium point. After the fault is cleared, the operating point will converge back to the post-fault equilibrium point, if the system is stable, or will drift away if the system is unstable. Assume the asymmetric stable points of the system are defined as set $A$. The basin stability samples the post-fault system state space and quantifies the likelihood that the system operating state returns back to set $A$ after perturbations. From the control theory point of view, the basin stability method numerically estimates the ROA of the post-fault system. In basin stability literatures [13], [21], the ROA is denoted as basin of attraction set $\mathcal{B}(A)$. By definition, basin stability is formulated as

$$
\begin{gathered}
S(\mathcal{B})=\int_{X} \chi_{\mathcal{B}}(X) \rho(X) d X, \\
\chi_{\mathcal{B}}(X)=\left\{\begin{array}{ll}
1 & \text { if }(X) \in \mathcal{B} \\
0 & \text { otherwise }
\end{array},\right.
\end{gathered}
$$

where $S(\mathcal{B}) \in[0,1]$ is the basin stability value. It equals 1 when the system is globally stable and equals 0 when the system is fully unstable. $X$ is a vector of system states that are disturbed at post-fault. $\mathcal{X}_{\mathcal{B}}(X)$ is an indicator function, which can justify the stability of $X . \rho(X)$ is a probability density function of the post-fault system states in the state space, $\int_{X} \rho(X) d x=1$.

\section{A. Implementation of basin stability method}

Applying the basin stability method to the elementary system model on transient stability, we consider the swing dynamic states and voltages of the generator are disturbed by the fault, which means at the post-fault initial moment (fault cleared), $X=\left(\theta, \omega, v_{c}, v_{f d}, \lambda_{f d}\right)$. States of the governor and turbine stay the same as the pre-fault values at the post-fault initial moment and oscillate in terms of the disturbed rotor angle $\theta$ and frequency $\omega$ during the post-fault simulation. For the test system in this work, we also assume that the post-fault system has the same configuration as the pre-fault system. Other configurations such as line losses can also be analyzed with the basin stability method in a future study of multi-bus systems. The probability density function $\rho(X)$ is set as a bounded uniform distribution in terms of $X$. The boundary of $\theta$ is chosen as $[-\pi,+\pi]$, which covers the entire $2 \pi$. The boundaries of $\omega, v_{c}, v_{f d}$ and $\lambda_{f d}$ are chosen as $\pm 20 \%$ from the per unit values at the stable equilibrium point. These boundaries are used because it is large enough to cover the most of the ROA of this system for the results in next section. In addition, generators usually will be tripped if the frequency or other states violate the limits. The uniform distribution of $X$ is assumed between the boundaries due to the fact that the probability distribution of the post-fault states depends on the distributions of possible faults and uncertainties. However, it is a lack of historical data to formulate the distributions of these rare events. Simple distributions of fault scenarios and uncertainties from renewable energy generations scenarios are used in [8], [9], and arbitrary transmission line fault distribution is used in [11]. In addition, the assumption of the uniform distribution of the postfault states can cover more potential disturbances in terms of 
the combinations of $\left(\theta, \omega, v_{c}, v_{f d}, \lambda_{f d}\right)$ than the assumptions of distributions of a finite number of fault types and uncertainty patterns is considered.

In this work, the time-domain simulation is used as the indicator function $\mathcal{X}_{\mathcal{B}}(X)$ because it can easily incorporate with high-order, detailed system models and provide accurate and reliable results. Lyapunov-based energy function methods is another branch of methods commonly used to justify the stability of the post-fault system; however, it is very hard to formulate the convex energy function and solve the unstable equilibrium points of the detailed system model such that it considers losses, generator's turbine, governor, exciter, and inverter with PLL and power and current controllers.

With above assumptions, the numerical implementation procedures of the basin stability method are as follows:

(1) Uniformly draw a number of $N$ random samples from the post-fault state space;

(2) Perform the time-domain simulations for all the samples selected and count the number of stable cases as $U$;

(3) Approximate the basin stability value numerically as $S(\mathcal{B}) \approx U / N$.

Because each sample is a repeated Bernoulli experiment, the Absolute Standard Error (ASE) is defined as

$$
e=\sqrt{S(\mathcal{B})(1-S(\mathcal{B}))} / \sqrt{N},
$$

A sufficient number of $N$ samples are needed to ensure the convergence of the proposed method on the elementary test system. To justify this number for the test system, we first define the penetration level as, $P_{P L}=P_{i} /\left(P_{i}+P_{m}\right)$, where $P_{i}$ and $P_{m}$ are the power generations from inverter and generator. Then, two groups of tests are taken with $P_{P L}=10 \%$ and $P_{P L}=$ $20 \%$, repectively. Each group consists of 10 sets of simulations. In each set, the above basin stability simulation procedures are repeatedly preformed with increasing numbers of samples $N$. The resulting basin stability values are shown in Fig. 4. From the results, both groups of experiments are successfully converged. Also in consideration of the tradeoff between the average ASE and average computational time of each set of simulations in Fig. 5, we chose $N=1.6 \times 10^{5}$ for the later simulations of this system. In this case, the ASE with $10 \%$ and $20 \%$ penetration is equal to 0.0012 and 0.0011 , respectively. All the simulations are performed with the help of an Nvidia Tesla P100 GPU. For $1.6 \times 10^{5}$ samples, the average program computation time is $106.9 \mathrm{~s}$. Therefore, the transient stability of the system can be investigated with basin stability framework in a timely fashion.

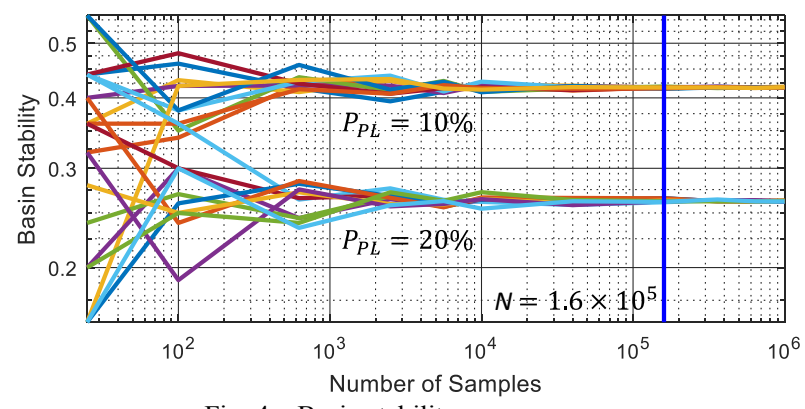

Fig. 4. Basin stability convergence.
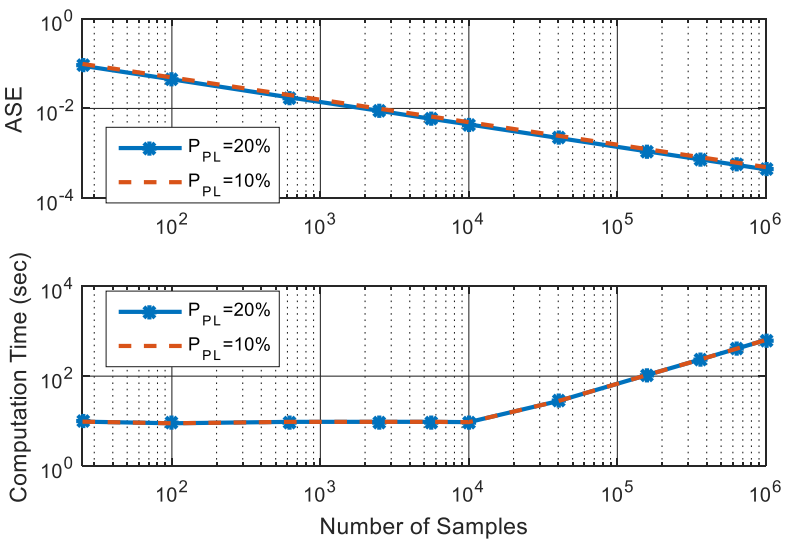

Fig. 5. Basin stability convergence.

\section{CASE STUdies}

\section{A. Basin stability plots at single penetration levels}

The estimated ROA of the generator in the elementary model that results from the basin stability method is shown in Fig. 6 with penetration level $P_{P L}$ at $10 \%$. The post-fault equilibrium point is at the center of the plot $\left(\theta_{0}, \omega_{0}\right)$. The $\mathrm{x}-$ axis is the generator rotor angle, which ranges from $\theta_{0}-\pi$ to $\theta_{0}+\pi$, and y-axis is the generator frequency from $\omega_{0}-0.2$ to $\omega_{0}+0.2$ in per unit value. The green area indicates the stable regions. Note that the post-fault operating points do not move on the plotted 2-D plot, but in the state space of the full-order inverter-generator model. This plot shows only the projection of the stability results in terms of $\theta$ and $\omega$ out of $X$. In each of the basin stability plots, $1.6 \times 10^{5}$ samples of simulations were performed. Fig. 7 shows the stable regions of the generator under penetration level $P_{P L}$ at $15 \%, 20 \%, 25 \%$ and $30 \%$, respectively. From subplots in Fig. 7 , it is obvious that the higher penetration level reduces the volume of the ROA. This indicates that the inverter penetration has a negative impact on the transient stability of the generator.

\section{B. Basin stability values at different penetration levels}

The ROA provides a visual representation of transient stability of the system. To quantify such properties, the basin stability value is numerically approximated as the number of stable samples $U$ over the number of total samples $N$. Under

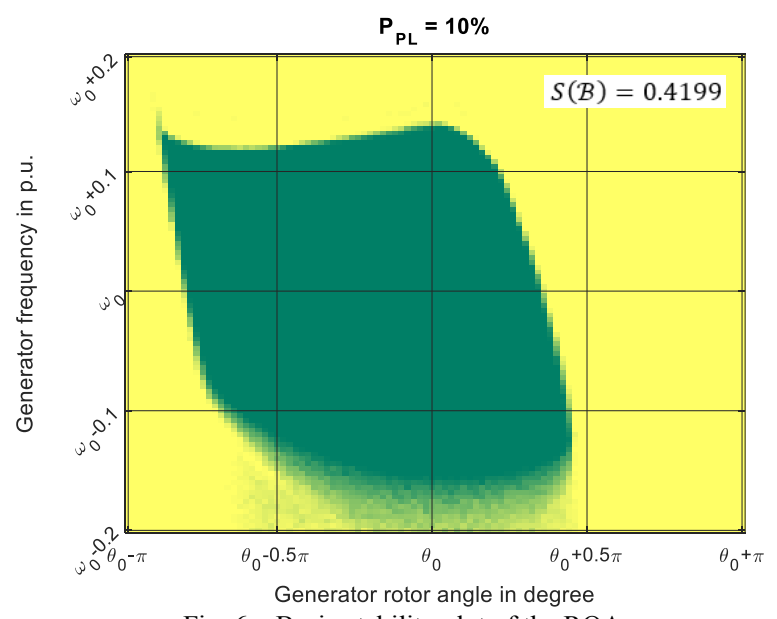

Fig. 6. Basin stability plot of the ROA 

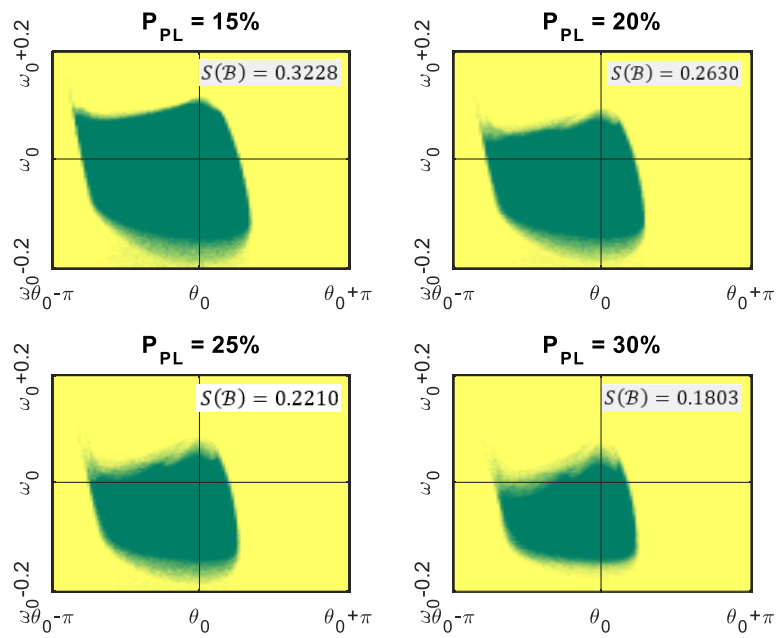

Fig. 7. Basin stability plots under different penetration levels.

the assumptions of the uniform distribution, it is proportional to the size of the green stable area over the whole area being investigated. In the following simulations, we gradually increased the penetration level $P_{P L}$, and calculated the corresponding basin stability value. The incremental step size of the $P_{P L}$ is $1 \%$. Fig. 8 shows the basin stability value versus the penetration level $P_{P L}$ under the default system parameters as the normal case. In the figure, the basin stability value is continuously decreasing when the $P_{P L}$ increases. This verifies the observations from pervious figures. The system turns fully unstable when $P_{P L}=36 \%$.

In addition to the normal case, the impact of different parameter settings to the transient stability with inverter penetrations is also studied and compared with the normal case. Fig. 9 shows how the different generator inertias $M$ effect the basin stability value. Two cases with $100 M$ and $0.01 M$ are compared with the normal case. From the plot, smaller inertia had negative impact on the basin stability, and surprisingly, the larger inertia does not yield much improvement of the system stability which will be further investigated.

Next, we modify the inverter current PI controller gains, $K_{P}$ and $K_{I}$ from the original value in the normal case. Both of them

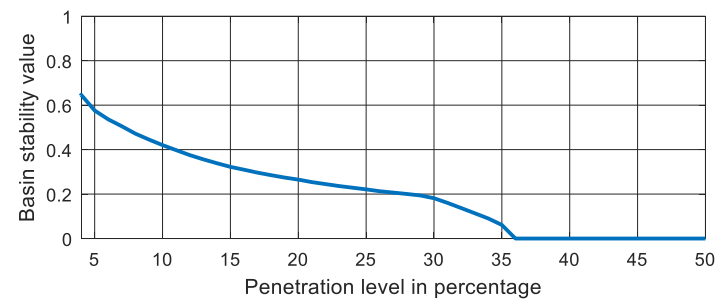

Fig. 8. Basin stability values under the normal case.

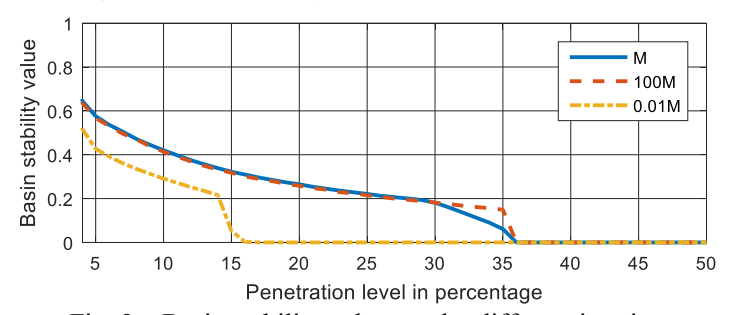

Fig. 9. Basin stability values under different inertias.

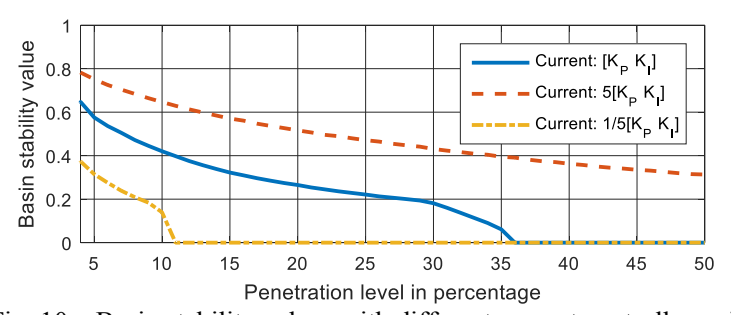

Fig. 10. Basin stability values with different current controller-gains.

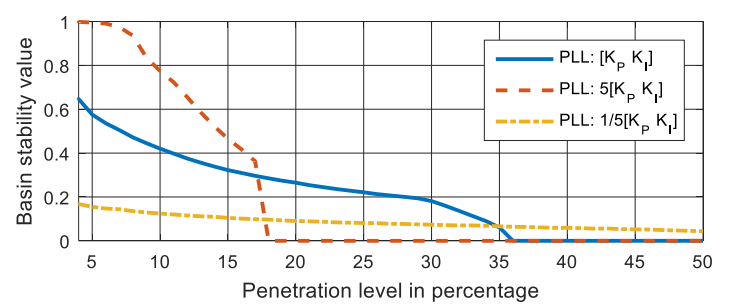

Fig. 11. Basin stability values with different PLL controller-gains.

are scaled by factors of 5 and $1 / 5$ in the simulation. From the results in Fig. 10, these controller-gains have a significant impact on the system stability at all penetration levels. Larger controllers' gains result in more stable systems. The point where the system becomes fully unstable also dramatically changed along with the changes of controller gains. These results indicate that aggressive inverter controller gains can improve the transient stability with respect to inverterinterfaced generations.

Lastly, the PLL has a major impact on the system stability, because it is responsible for synchronizing the inverter output ac voltage with the grid. To explore this, the PI controller-gains of PLL are also scaled by factors of 5 and 1/5. Results in Fig. 11 shows interesting results. The higher gains of PLL can increase the stability at very low penetration levels, but the basin stability drops more quickly compared with the normal case as the penetration level increases. The lower gains have a flat curve where they reduce the system stability at low penetration levels and increase the stability when the penetration level passes $35 \%$.

\section{The impact of inverter penetration with a modified IEEE 9-bus system model}

The IEEE 9-bus system is modified by replacing the generator on Bus 3 with the scalable inverter model in Fig. 12. The power injection from generators at Bus 1 and 2 are set to be equal, and the inverter generation is defined by the penetration level. The Matpower toolbox is used to solve the power-flow [22]. The same assumptions of the basin stability method are also applied to this system. The basin stability values of generators at Bus 1 and 2 are shown in Fig. 13. From the results, both basin stability values decrease when penetration level increase. It further confirms the conclusion that, increasing penetrations of the inverter-interfaced generation has a clear negative impact on the generator transient stability.

\section{CONCLUSION AND FUTURE WORKS}

In this study, we investigated the impact of inverterinterfaced generations on the generator transient stability. A scalable inverter model is used to accomplish the systematical 


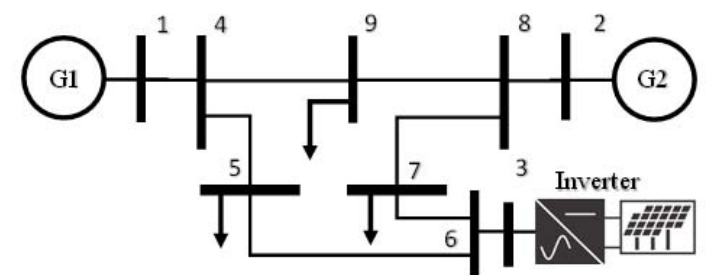

Fig. 12. The Modified IEEE 9-bus system model.

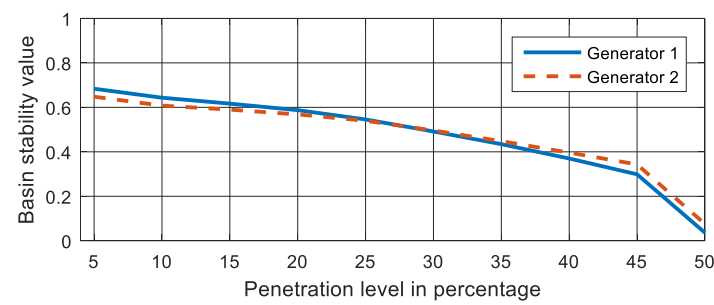

Fig. 13. Basin stability values of two generators in the modified IEEE

scaling of inverter parameters for these simulations. To explicitly demonstrate the variations of the system stability against different penetration levels, a basin stability method is used to estimate the ROA and quantify the transient stability. The proposed method is able to capture the nonlinear interaction among inverters and generators. Moreover, such a sample-based approach can take advantage of recent parallel computing techniques. From the simulation results, we concluded that the penetrations of inverter-interfaced generations under the conventional grid-following control scheme could have a negative impact on the system transient stability. However, additional design efforts, such as tuning of controllers' gains, can improve the stability at different penetration levels. This knowledge contains important basic principles for understanding the impact of inverter-interfaced generation on transient stability in more complex multi-bus system analysis.

Based on the lessons learned, future research directions are 1) find the possible reasons of the evolvement of the ROA under different cases, 2) apply simulations to larger systems and check how system topology affects the stability, 3) develop new control methods for inverters to improve the transient stability of the system.

\section{ACKNOWLEDGEMENT}

Funding provided by U.S. Department of Energy Office of Energy Efficiency and Renewable Energy Solar Energy Technologies Office. The views expressed in the article do not necessarily represent the views of the DOE or the U.S. Government. The U.S. Government retains and the publisher, by accepting the article for publication, acknowledges that the U.S. Government retains a nonexclusive, paid-up, irrevocable, worldwide license to publish or reproduce the published form of this work, or allow others to do so, for U.S. Government purposes.

\section{REFERENCES}

N. W. Miller, "Keeping It Together: Transient Stability in a World of Wind and Solar Generation," IEEE Power Energy Mag., vol. 13, no. 6 , pp. 31-39, 2015.

[2] V. Purba, S. V Dhople, S. Jafarpour, F. Bullo, and B. B. Johnson, "Reduced-order structure-preserving model for parallel-connected three-phase grid-tied inverters," 2017 IEEE 18th Work. Control Model. Power Electron., pp. 1-7, 2017.

[3] Y. Lin, B. Johnson, and V. Gevorgian, "Stability Assessment of a System Comprising a Single Machine and Inverter with Scalable Ratings," NAPS 2017 - 49th North Am. Power Symp. Proc., 2017.

[4] K. Kawabe and K. Tanaka, "Impact of Dynamic Behavior of Photovoltaic Power Generation Systems on Short-Term Voltage Stability," Power Syst. IEEE Trans., vol. 30, no. 6, pp. 3416-3424, 2015.

[5] B. Tamimi, C. Canizares, and K. Bhattacharya, "System stability impact of large-scale and distributed solar photovoltaic generation: The case of Ontario, Canada," IEEE Trans. Sustain. Energy, vol. 4, no. 3, pp. 680-688, 2013.

[6] D. K. Dheer, N. Soni, and S. Doolla, "Small signal stability in microgrids with high penetration of power electronics interfaced sources," IECON Proc. (Industrial Electron. Conf., no. 1, pp. 2272 $2278,2014$.

[7] D. K. Dheer, S. Doolla, and A. K. Rathore, "Small Signal Modeling and Stability Analysis of a Droop Based Hybrid AC/DC Microgrid," IECON Proc. (Industrial Electron. Conf., no. c, pp. 3775-3780, 2016.

[8] K. J. Timko, A. Bose, and P. M. Anderson, "Monte Carlo Simulation of Power System Stability," IEEE Trans. Power Appar. Syst., vol. PAS-102, no. 10, pp. 3453-3459, 1983.

[9] P. N. Papadopoulos and J. V. Milanovic, "Probabilistic Framework for Transient Stability Assessment of Power Systems with High Penetration of Renewable Generation," IEEE Trans. Power Syst., vol. 32, no. 4, pp. 3078-3088, 2017.

[10] J. C. O. Mello, M. V. F. Pereira, and a. M. Leite da Silva, "Evaluation of reliability worth in composite systems based on pseudo-sequential Monte Carlo simulation," IEEE Trans. Power Syst., vol. 9, no. 3, pp. 1318-1326, 1994

[11] T. Guo and J. V Milanovic, "Probabilistic Framework for Assessing the Accuracy of Data Mining Tool for Online Prediction of Transient Stability," IEEE Trans. Power Syst., vol. 29, no. 1, pp. 377-385, 2014.

[12] R. Billinton and P. R. S. Kuruganty, "Probabilistic Assessment of Transient Stability in a Practical Multimachine System," IEEE Trans. Power Appar. Syst., vol. PAS-100, no. 7, pp. 3634-3641, 1981.

[13] P. J. Menck, J. Heitzig, N. Marwan, and J. Kurths, "How basin stability complements the linear-stability paradigm," Nat. Phys., vol. 9, no. 2, pp. 89-92, 2013.

[14] P. J. Menck, J. Heitzig, J. Kurths, H. J. Schellnhuber, and P. J. Menck, "How dead ends undermine power grid stability," Nat. Commun., vol. 5, pp. 1-8, 2014

[15] P. Ji and J. Kurths, "Basin stability of the Kuramoto-like model in small networks," Eur. Phys. J. Spec. Top., vol. 2491, pp. 2483-2491, 2014.

[16] K. Schmietendorf, J. Peinke, R. Friedrich, and O. Kamps, "Selforganized synchronization and voltage stability in networks of synchronous machines," Eur. Phys. J. Spec. Top., vol. 2592, pp. 2577-2592, 2014

[17] H. Kim, S. H. Lee, and P. Holme, "Community consistency determines the stability transition window of power-grid nodes," New J. Phys., vol. 17, no. 11, p. 113005, 2015.

[18] Z. Liu and Z. Zhang, "Quantifying Transient Stability of Generators by Basin Stability and Kuramoto-like Models," NAPS 2017 - 47th North Am. Power Symp. Proc., 2017.

[19] Z. Liu, X. He, Z. Ding, and Z. Zhang, "A Basin Stability-Based Metric for Ranking the Transient Stability of Generators," IEEE Trans. Ind. Informatics, in press.

[20] P. Kundur, "Power System Stability And Control." McGraw-hill, New York, 1994.

[21] P. Schultz, P. J. Menck, J. Heitzig, and J. Kurths, "Potentials and limits to basin stability estimation," New J. Phys., vol. 19, no. 2, 2017.

[22] R. D. Zimmerman, C. E. Murillo-Sanchez, and R. J. Thomas, "MATPOWER: Steady-State Operations, Planning, and Analysis Tools for Power Systems Research and Education," IEEE Trans. Power Syst., vol. 26, no. 1, pp. 12-19, 2011. 